\title{
Nonlinearly coupled upper-hybrid and magnetoacoustic waves in collisional magnetoplasmas
}

\author{
P. K. Shukla, ${ }^{1, *}$ B. Eliasson, ${ }^{1}$ and L. Stenflo ${ }^{2}$ \\ ${ }^{1}$ Institut für Theoretische Physik IV, Fakultät für Physik und Astronomie, Ruhr-Universität Bochum, D-44780 Bochum, Germany \\ ${ }^{2}$ Department of Plasma Physics, Umeå University, SE-90187 Umeå, Sweden
}

(Received 10 June 2003; published 23 December 2003)

\begin{abstract}
The nonlinear interaction between large amplitude electrostatic upper-hybrid (UH) waves and magnetoacoustic perturbations in a collisional magnetoplasma is considered. For this purpose, we present a nonlinear Schrödinger equation for the UH wave envelope in the presence of the electron density, electron temperature, and compressional magnetic field perturbations of the dispersive magnetoacoustic waves, and derive an equation for the latter taking into account the combined influence of the UH ponderomotive force and the differential electron Joule heating in the UH wave electric field. The coupled equations are employed to investigate modulational instabilities of a constant amplitude UH wave, and the density profile modifications due to the $\mathrm{UH}$ ponderomotive and thermal forces. It is found that the thermal instability has its maximum at a wavelength half the one of the UH wave, and a numerical solution of the coupled time-dependent system of equations reveals that localized density cavities are also created with a spacing approximately half the wavelength of the pump wave.
\end{abstract}

DOI: 10.1103/PhysRevE.68.067401

PACS number(s): 52.35.Fp, 52.35.Mw, 52.35.Sb, 94.20.Ji

In the past, a number of papers [1-7] have considered the nonlinear coupling between upper-hybrid (UH) waves and low-frequency electrostatic and electromagnetic waves [8-11], taking into account the $\mathrm{UH}$ wave ponderomotive force. It has been found that a large amplitude UH wave can decay into a circularly or elliptically polarized electromagnetic wave and a host of low-frequency waves (especially lower-hybrid waves). On the other hand, the UH wave can also be modulated by nonresonant density and magnetic field perturbations. The resulting modulational instabilities can be responsible for the formation of localized UH envelope solitons, accompanied with density humps or dips depending on the group dispersion of the UH wave.

However, in a collisional magnetoplasma, there is a thermal nonlinearity associated with the electron Joule heating [12] in the UH electric field. The electron heating causes electron temperature fluctuations which are coupled with the plasma density perturbations. When the modulation frequency is smaller than the electron-ion collision frequency, the electron Joule heating nonlinearity dominates over the ponderomotive force nonlinearity. The Joule heating is one of the candidates for the creation of striations [13] via the combined action of Joule heating and the oscillating twostream instability $[14,15]$, or via self-focusing of electromagnetic waves combined with the trapping of upper-hybrid waves and Joule heating [16-18]. Striations are important for the interaction of magnetized space plasmas with electromagnetic waves [19-21].

In this Brief Report, we consider the amplitude modulation of a large amplitude UH wave by dispersive magnetoacoustic waves, taking into account the combined effects of the UH wave ponderomotive force and the differential electron Joule heating on the magnetoacoustic wave motions. We derive a coupled system of equations which exhibit nonlinear

\footnotetext{
*Also at the Department of Plasma Physics, Umeå University, SE-90187 Umeå, Sweden.
}

coupling between the UH wave electric field, the compressional magnetic field perturbation, and the electron temperature fluctuation. The coupled mode equations are then used to study modulational instabilities in the highly collisional regime. A nonlinear dispersion relation for the growth rates is presented and solved numerically for parameters typical for the Earth's ionosphere. We also perform simulations of our nonlinear equations, and apply our results to the Earth's lower ionosphere where interparticle collisions are dominant. We thus have a self-consistent picture of the creation of density striations by the UH waves, contrary to the preexisting density striations assumed in previous investigations.

Let us consider the nonlinear propagation of electrostatic UH waves in an electron-ion plasma which is immersed in an external magnetic field $B_{0} \hat{\mathbf{z}}$, where $B_{0}$ is the magnitude of the external magnetic field, and $\hat{\mathbf{z}}$ is the unit vector along the $z$ axis. The UH wave electric field is $\mathbf{E}=\left(E_{\perp} \hat{\mathbf{x}}+E_{z} \hat{\mathbf{z}}\right) \exp (i \mathbf{k} \cdot \mathbf{r}-i \omega t)$, where the subscripts $\perp$ and $z$ stand for the perpendicular and parallel (to $\hat{\mathbf{z}}$ ) components, respectively. The frequency $\omega$ and the wave vector $\mathbf{k}$ $\left(=k_{\perp} \hat{\mathbf{x}}+k_{z} \hat{\mathbf{z}}\right)$ are, if $k_{z} \ll k_{\perp}$, related by

$$
\omega^{2} \approx \omega_{H}^{2}+3 \alpha k_{\perp}^{2} v_{t e}^{2}-\left(k_{z}^{2} / k_{\perp}^{2}\right)\left[\left(\omega_{p e}^{2} \omega_{c e}^{2}\right) / \omega_{H}^{2}\right]
$$

where $\omega_{H}^{2}=\omega_{p e}^{2}+\omega_{c e}^{2}, \omega_{p e}$ is the electron plasma frequency, $\omega_{c e}$ is the electron gyrofrequency, $\alpha=\left(1-3 \omega_{c e}^{2} / \omega_{p e}^{2}\right)^{-1}$, $v_{t e}=\left(T_{e} / m_{e}\right)^{1 / 2}$ is the electron thermal speed, $e$ is the magnitude of the electron charge, $m_{e}$ is the electron mass, $T_{e}$ is the electron temperature, and $c$ is the speed of light in vacuum.

The nonlinear interaction between the UH waves and lowfrequency (in comparison with $\omega_{H}$ ) magnetoacoustic waves in magnetized plasmas leads to an envelope of waves, which is governed by 


$$
\begin{aligned}
i\left(\partial_{t}+\right. & \left.\Gamma_{u h}+\mathbf{v}_{g} \cdot \nabla_{\perp}\right) E_{\perp}+\frac{3 \alpha v_{t e}^{2}}{2 \omega_{H 0}} \nabla_{\perp}^{2} E_{\perp}-\frac{\omega_{p 0}^{2}}{2 \omega_{H 0}} \frac{n_{e 1}}{n_{e 0}} E_{\perp} \\
& -\frac{\omega_{c 0}^{2}}{\omega_{H 0}} \frac{B_{1}}{B_{0}} E_{\perp}=0,
\end{aligned}
$$

where $\mathbf{v}_{g}=3 \alpha\left(v_{t e}^{2} / \omega_{H 0}\right) \mathbf{k}_{\perp}$ is the group velocity of the almost perpendicularly propagating UH waves [viz. without the last term on the right-hand side of Eq. (1)], $\omega_{H 0}=\left(\omega_{p 0}^{2}\right.$ $\left.+\omega_{c 0}^{2}\right)^{1 / 2}, \quad \omega_{p 0}=\left(4 \pi n_{0} e^{2} / m_{e}\right)^{1 / 2}, \quad \omega_{c 0}=e B_{0} / m_{e} c, \quad n_{e 1}$ $\left(\ll n_{0}\right)$, and $B_{1}\left(\ll B_{0}\right)$ are small electron number density and compressional magnetic field perturbations associated with dispersive magnetoacoustic perturbations, and $n_{0}$ is the unperturbed electron number density. For completeness we have also introduced the phenomenological damping operator $\Gamma_{u h}$ to account for the damping of the UH wave due to collisions.

The UH wave envelope exerts a low-frequency ponderomotive force on the dispersive magnetoacoustic waves. This ponderomotive force of the $\mathrm{UH}$ waves acts mainly on the electron fluid, but is transmitted to the ions via the electromagnetic fields of the magnetoacoustic waves. The electron ponderomotive force is

$$
\mathbf{F}_{e}=-m_{e}\left\langle\mathbf{v}_{e}^{H} \cdot \nabla \mathbf{v}_{e}^{H}\right\rangle \equiv \mathbf{F}_{e \perp}+F_{e z} \hat{\mathbf{z}},
$$

where $\mathbf{v}_{e}^{H}$ is the electron quiver velocity in the UH wave electric field and the angular bracket denotes averaging over the UH wave period $\left(2 \pi / \omega_{H 0}\right)$. We have [2]

$$
\mathbf{F}_{e \perp}=-\left(e^{2} / 4 m_{e}\right)\left(\omega_{H 0}^{2} / \omega_{p 0}^{4}\right) \nabla_{\perp}\left|E_{\perp}\right|^{2}
$$

and

$$
F_{e z}=-\left[e^{2} /\left(4 m_{e} \omega_{p 0}^{2}\right)\right] \partial_{z}\left|E_{\perp}\right|^{2} .
$$

We shall now present the relevant equations for onedimensional magnetoacoustic waves in the presence of the UH ponderomotive force and the differential electron Joule heating in the UH wave electric field, considering wave propagation along the $x$ axis in a quasineutral plasma, and using standard notations where, for example, $n_{1}\left(\approx n_{e 1}\right)$ is the ion density perturbation and $v_{x}$ is the $x$ component of the ion fluid velocity. We then have the ion continuity equation

$$
\partial_{t} n_{1}+n_{0} \partial_{x} v_{x}=0
$$

the ion momentum equation

$$
\begin{aligned}
\partial_{t} v_{x}= & -\frac{B_{0}}{4 \pi n_{0} m_{i}} \partial_{x} B_{1}-\frac{T_{e}+T_{i}}{n_{0} m_{i}} \partial_{x} n_{1}-\frac{1}{m_{i}} \partial_{x} T_{1} \\
& -\frac{e^{2}}{4 m_{e} m_{i}} \frac{\omega_{H 0}^{2}}{\omega_{p 0}^{4}} \partial_{x}\left|E_{\perp}\right|^{2},
\end{aligned}
$$

the energy equation

$$
3 \partial_{t} T_{1}-\frac{T_{e}+T_{i}}{n_{0}} \partial_{t} n_{1}=\frac{\nu_{e}}{4 \pi n_{0}} \frac{\omega_{H 0}^{2}}{\omega_{p 0}^{2}}\left|E_{\perp}\right|^{2},
$$

and the $y$ component of Ampere's law

$$
\partial_{x} B_{1}=(4 \pi / c) n_{0} e v_{e y},
$$
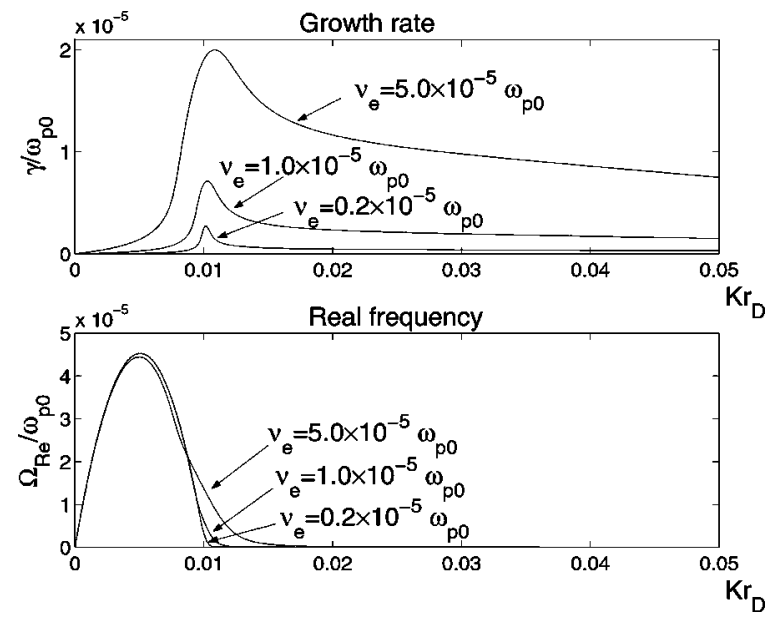

FIG. 1. The growth rate $\gamma$ (upper panel) and real frequency $\Omega_{R e}$ (lower panel) as a function of the wave number $K$; the electric field has initial wave number $0.005 r_{D}^{-1}$ and amplitude $E_{\perp 0}$ $=0.041 \sqrt{16 \pi n_{0}\left(T_{e}+T_{i}\right)}$.

where $v_{e y}$ is determined from

$$
\left(\partial_{t}+\nu_{e}\right) v_{e y}=-\left(e / m_{e}\right) E_{y}+\omega_{c e} v_{x} .
$$

The $y$ component of the dispersive magnetoacoustic wave electric field is found from Faraday's law

$$
\partial_{x} E_{y}=-c^{-1} \partial_{t} B_{1} .
$$

We note that in a quasineutral plasma, the $x$ component of the electron and ion fluid velocities involved in the magnetoacoustic waves are equal, while $v_{i y}=0$. Equation (7) has been obtained by adding the $x$ component of the ion and inertialess electron momentum equations. This equation includes the sum of the electron and ion temperature perturbations $T_{1}$ and the UH ponderomotive force (the last term on the right-hand side). The right-hand side in Eq. (8) is the differential electron Joule heating in the UH wave electric field. We have assumed here that the magnetoacoustic wave frequency is much larger than $\nu_{e} \rho_{e}^{2}\left|\partial_{x}^{2}\right|$ and $\nu_{i} \rho_{i}^{2}\left|\partial_{x}^{2}\right|$, where $\rho_{e}\left(\rho_{i}\right)$ is the electron (ion) gyroradius. The electron-ion (ion-ion) collision frequency is denoted by $\nu_{e}\left(\nu_{i}\right)$.

Combining Eqs. (9)-(11), we have

$$
\partial_{t} \tilde{N}=\left[\partial_{t}\left(1-\lambda_{e}^{2} \partial_{x}^{2}\right)-\nu_{e} \lambda_{e}^{2} \partial_{x}^{2}\right] \widetilde{B}
$$

where $\widetilde{N}=n_{1} / n_{0}$ and $\widetilde{B}=B_{1} / B_{0}$. Equation (12) shows that the frozen-in-field relation is broken due to the finite electron inertial effect in Eq. (10). Here, $\lambda_{e}=c / \omega_{p 0}$ is the electron skin depth. Hence, our governing equations are

$$
i\left(\partial_{t}+\Gamma_{u h}+v_{g} \partial_{x}\right) \widetilde{E}+\frac{3 \alpha v_{t e}^{2}}{2 \omega_{H 0}} \partial_{x}^{2} \widetilde{E}-\frac{\omega_{p 0}^{2}}{2 \omega_{H 0}} \widetilde{N} \widetilde{E}-\frac{\omega_{c 0}^{2}}{\omega_{H 0}} \widetilde{B} \widetilde{E}=0,
$$

$$
\left(\partial_{t}^{2}-C_{a}^{2} \partial_{x}^{2}\right) \widetilde{N}-V_{A}^{2} \partial_{x}^{2} \widetilde{B}-C_{a}^{2} \partial_{x}^{2} \widetilde{T}=C_{a}^{2} \partial_{x}^{2}|\widetilde{E}|^{2}
$$

and

$$
\partial_{t} \widetilde{T}-\left(\partial_{t} \tilde{N} / 3\right)=\left(4 \nu_{e} / 3\right)|\widetilde{E}|^{2}
$$



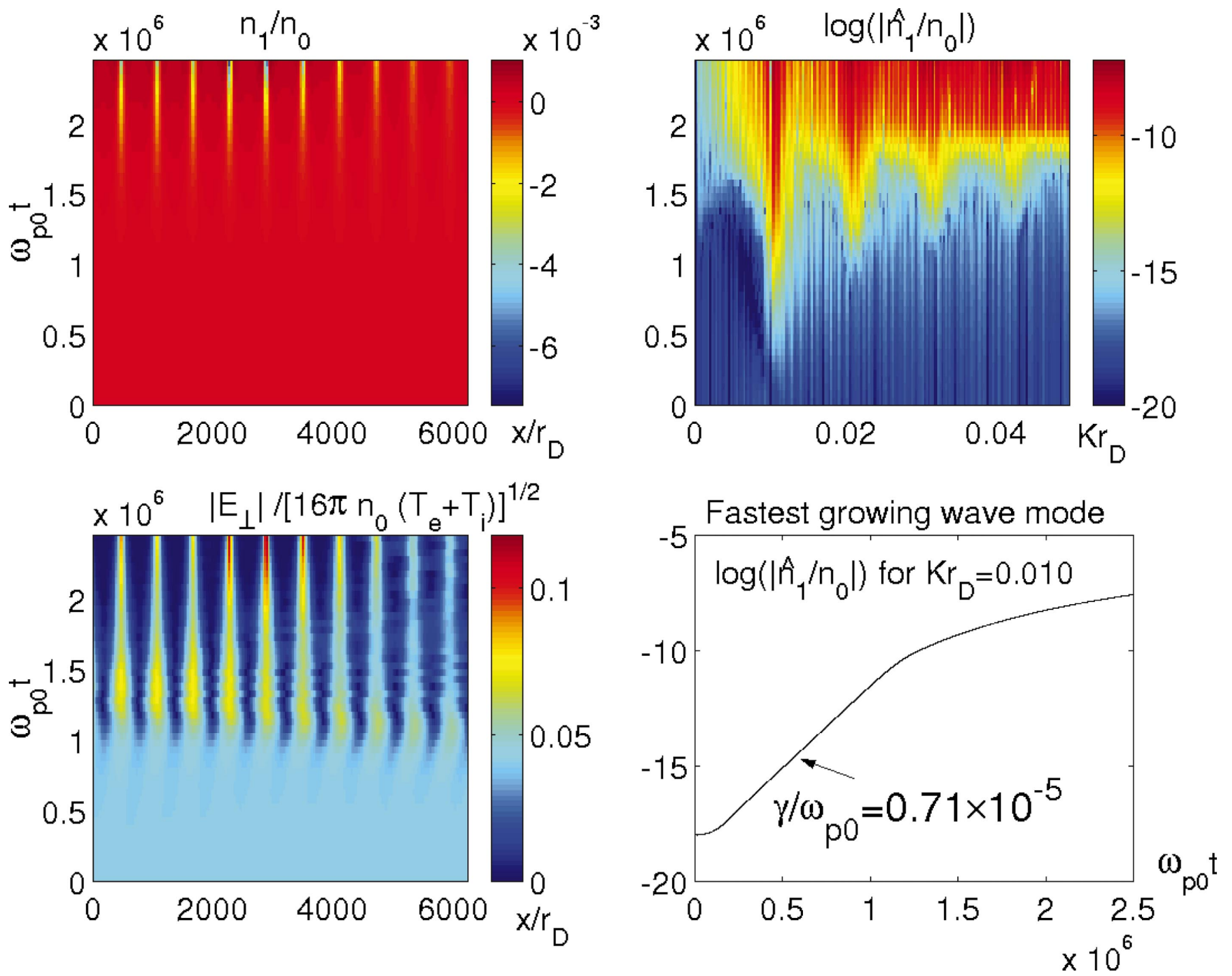

FIG. 2. (Color) The time development of the density $n_{1}$ (upper left panel), the wave number spectrum (upper right panel), the highfrequency electric field (lower left panel), and the fastest growing wave mode (lower right panel); the electric field has initially the wave number $0.005 r_{D}^{-1}$ and amplitude $E_{\perp 0}=0.041 \sqrt{16 \pi n_{0}\left(T_{e}+T_{i}\right)}$.

where $\quad \widetilde{T}=T_{1} /\left(T_{e}+T_{i}\right), \quad \widetilde{E}=\left(\omega_{H 0} / \omega_{p 0}\right) E_{\perp} /$ $\sqrt{16 \pi n_{0}\left(T_{e}+T_{i}\right)}, \quad C_{a}=\left[\left(T_{e}+T_{i}\right) / m_{i}\right]^{1 / 2}, \quad$ and $V_{A}$ $=B_{0} / \sqrt{4 \pi n_{0} m_{i}}$.

Equations (13)-(15) together with Eq. (12) are generalized Zakharov equations for a collisional magnetoplasma. They govern the dynamics of nonlinearly coupled UH and dispersive magnetoacoustic modes.

Following the standard procedure [22], we now derive the nonlinear dispersion relation for investigating modulational instabilities of a constant amplitude UH pump $E_{\perp 0} \exp \left(-i \omega_{0} t+i k_{x 0} x\right)+$ complex conjugate. Thus, we decompose $\widetilde{E}$ as a sum of the pump and the two UH sidebands $\left(\omega_{ \pm}, k_{x \pm}\right)$, where $\omega_{ \pm}=\Omega \pm \omega_{0}$ and $k_{x \pm}=K \pm k_{x 0}$. The frequency and the wave number of the magnetoacoustic wave are denoted by $\Omega$ and $K$, respectively. We then find from Eqs. (13) to (15) that

$$
\Omega^{2}-\Omega_{m}^{2}=\omega_{H 0}^{2} K^{2} C_{a}^{2}\left(1+i \frac{4 \nu_{e}}{3 \Omega}\right)\left(1+\frac{2 \omega_{c 0}^{2}}{\omega_{p 0}^{2} X}\right) \sum_{+,-} \frac{W}{D_{ \pm}}
$$

where $\quad X=1+K^{2} \lambda_{e}^{2}\left(1+i \nu_{e} / \Omega\right), \quad \Omega_{m}^{2}=(4 / 3) K^{2} C_{a}^{2}$ $+K^{2} V_{A}^{2} / X, W=E_{\perp 0}^{2} / 16 \pi n_{0}\left(T_{e}+T_{i}\right)$, and $D_{ \pm}= \pm 2 \omega_{H 0}(\Omega$ $-K v_{g 0} \mp \delta$ ), with $\delta=3 K^{2} v_{t e}^{2} \alpha / 2 \omega_{H 0} \mp i \nu_{u h}$, where $\nu_{u h}$ is the Fourier transformed damping operator $\Gamma_{u h}$. Equation (16), which generalizes the corresponding dispersion relation of Ref. [23], can be rewritten as

$$
\begin{aligned}
& \left(\Omega^{2}-\Omega_{m}^{2}\right)\left[\left(\Omega-K v_{g 0}\right)^{2}-\delta^{2}\right] \\
& \quad=\omega_{H 0} \delta K^{2} C_{a}^{2}\left(1+i \frac{4 \nu_{e}}{3 \Omega}\right)\left(1+\frac{2 \omega_{c 0}^{2}}{\omega_{p 0}^{2} X}\right) W
\end{aligned}
$$

We have solved the dispersion relation (17) numerically for the wave number $k_{x 0}=0.005 r_{D}^{-1}$, the pump strength $E_{\perp 0}=0.041 \sqrt{16 \pi n_{0}\left(T_{e}+T_{i}\right)}$, and for the three different collision frequencies $\nu_{e}=0.2 \times 10^{-5} \omega_{p 0}, \nu_{e}=1.0 \times 10^{-5} \omega_{p 0}$, and $\nu_{e}=5 \times 10^{-5} \omega_{p 0}$; see Fig. 1. Here $r_{D}\left(=v_{t e} / \omega_{p 0}\right)$ is the electron Debye radius. We used the ion to electron mass ratio $m_{i} / m_{e}=29500$ valid for oxygen atoms and the ion to electron temperature ratio $T_{i} / T_{e}=0.5$. The electron temperature 


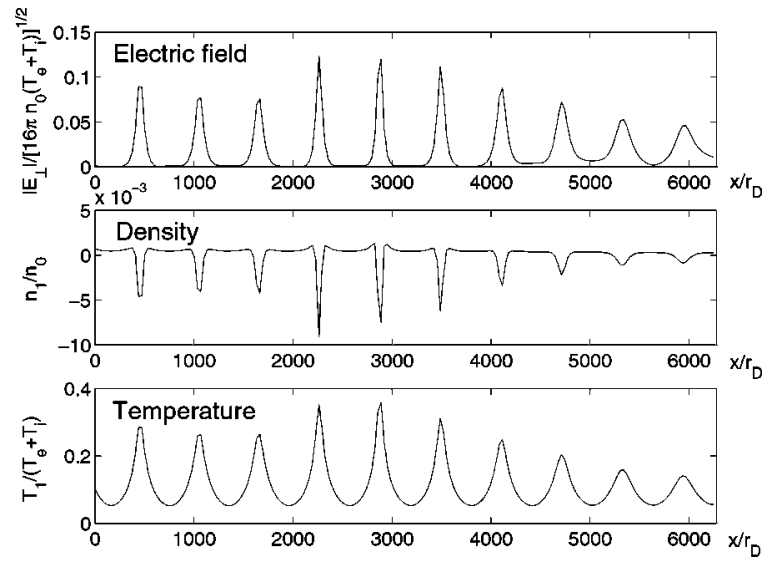

FIG. 3. The electric field, density, and temperature at time $t$ $=2.5 \times 10^{6} \omega_{p 0}^{-1}$, as a function of $x$, forming a "grid" of standing solitary wave solutions.

$T_{e}$ is $1500 \mathrm{~K}$. The ratio between the electron gyro and electron plasma frequencies is 0.25 . Thus the $\mathrm{UH}$ wave frequency is much larger than $\nu_{u h}$, thereby neglecting the collisional damping effect on the UH wave envelope. The above parameters are representative of the Earth's $F$ region. One can see (upper panel) that the instability with the maximum growth rate $\gamma$ at the wave number $\approx 2 k_{x 0}=0.01 r_{D}^{-1}$ increases strongly with increasing collision frequency. For the same wave number, the real part of the frequency is smaller or of the same order as the growth rate; thus these modes can be regarded as almost purely growing with wave numbers twice the pump wave numbers.

In order to assess the results and to go beyond the linear analysis of the system, we solved the time-dependent generalized Zakharov system (12)-(15) directly for the case $\nu_{e}$ $=1.0 \times 10^{-5} \omega_{p 0}$, with a constant-envelope initial condition on the $\mathrm{UH}$ wave electric field, $E_{t=0}=E_{\perp 0}$ with $E_{\perp 0}$ $=0.041 \sqrt{16 \pi n_{0}\left(T_{e}+T_{i}\right)}$. The numerical results are displayed in Figs. 2 and 3. In the left upper and lower panels of Fig. 2, respectively, the density and electric field envelopes are shown. In order to study the evolution of the wave number spectrum, the density was Fourier transformed in $x$ space and the time-dependent amplitude of the Fourier components are depicted in the right upper panel of Fig. 2. The timedependent amplitude of the fastest growing wave mode is exhibited in the lower right panel of Fig. 2. From the upper right panel, one can see that the fastest growing waves ap- pear at $K r_{D} \approx 0.01$, which was predicted from the linear analysis displayed in the upper panel of Fig. 1, where the maximum growth rate $\gamma / \omega_{p 0} \approx 0.71 \times 10^{-5}$ agrees well with the growth rate of the fastest growing mode obtained in the numerical simulation, displayed in the lower right panel of Fig. 2. After time $t=1.2 \times 10^{6} \omega_{p 0}^{-1}$, the exponential growth saturates and the density fluctuations and electric field envelopes become localized to an equidistant "grid," as can be seen in the left panels of Fig. 2. In Fig. 3, the $x$ dependence of the UH electric field envelope, plasma density fluctuation, and temperature perturbation are displayed at time $t$ $=2.5 \times 10^{6} \omega_{p 0}^{-1}$. At this time, the solution has reached a fully nonlinear stage; large and localized UH electric field envelope amplitudes are correlated with localized density depletions and temperature maxima; see the upper, middle and lower panels of Fig. 3, respectively.

In summary, we have considered the nonlinear propagation of large amplitude UH electrostatic waves in a collisional magnetoplasma. It is shown that the nonlinear coupling between the UH waves and dispersive magnetoacoustic waves generates an envelope of UH waves whose dynamics is governed by the Schrödinger equation (13). The ponderomotive and thermal forces of the UH wave envelope reinforces the plasma density, compressional magnetic field, and electron temperature perturbations. We thus have a selfconsistent description of the nonlinear interaction between the UH and driven magnetoacoustic perturbations in a collisional magnetoplasma. Our derived mode coupling equations have been employed for investigating modulational instabilities as well as UH envelope solitons consisting of localized UH wave envelopes and localized density and temperature perturbations. These perturbations were found to be spaced with a distance approximately half the wavelength of the pump upper-hybrid wave. The present analytical and simulation results should help to understand the nonlinear phenomena that are introduced by large amplitude UH waves in the $F$ region of the Earth's ionosphere where meter scale size density striations and temperature filaments are frequently observed [13].

This work was partially supported by the Deutsche Forschungsgemeinschaft through the Sonderforschungsbereich 591 as well as by the Swedish Research Council through Contract No. 621-2001-2274.
[1] A.N. Kaufman and L. Stenflo, Phys. Scr. 11, 269 (1975).

[2] M. Porkolab and M.V. Goldman, Phys. Fluids 19, 872 (1976).

[3] M.Y. Yu and P.K. Shukla, Plasma Phys. 19, 889 (1977).

[4] P.K. Shukla and M.Y. Yu, Phys. Rev. Lett. 49, 696 (1982).

[5] P.J. Christiansen et al., Phys. Rev. Lett. 46, 1333 (1981).

[6] L. Stenflo, Phys. Rev. Lett. 48, 1441 (1982); 48, 1870 (1982).

[7] R.P. Sharma and P.K. Shukla, Phys. Fluids 26, 87 (1983).

[8] G. Murtaza and P.K. Shukla, J. Plasma Phys. 31, 423 (1984); L. Stenflo and P.K. Shukla, Planet. Space Sci. 40, 473 (1992).

[9] B. Eliasson and P.K. Shukla, Phys. Lett. A 312, 91 (2003).

[10] W. Scales and H. Xi, Phys. Scr. T84, 184 (2000).

[11] S.P. Kuo, Phys. Lett. A 307, 244 (2003).
[12] L. Stenflo, J. Geophys. Res. [Atmos.] 90, 5355 (1985).

[13] R. Bharuthram et al., IEEE Trans. Plasma Sci. 20, 803 (1992).

[14] K.B. Dysthe et al., Phys. Scr. T2/2, 548 (1982).

[15] K.B. Dysthe et al., Phys. Fluids 26, 146 (1983).

[16] V.V. Vas'kov et al., Geomagn. Aeron. 24, 350 (1984).

[17] A.V. Gurevich et al., Phys. Rev. Lett. 75, 2622 (1995).

[18] A.V. Gurevich et al., Phys. Lett. A 239, 385 (1998).

[19] A.V. Gurevich et al., Phys. Lett. A 231, 97 (1997).

[20] E. Mjølhus, J. Plasma Phys. 29, 195 (1983).

[21] E. Mjølhus, J. Plasma Phys. 58, 747 (1997).

[22] P.K. Shukla and L. Stenflo, Phys. Fluids 29, 2479 (1986).

[23] L. Stenflo, Phys. Scr. T30, 166 (1990). 